\title{
Serum Macrophage Migration Inhibitory Factor Levels in Patients with Ischemic Stroke in Iraqi Populations
}

\author{
Manal M. Kadhim ${ }^{1}$, Ali S.Shakir ${ }^{2}$ \\ ${ }^{1}$ Professor of Clinical Immunology Department of Medical Microbiology, College of Medicine, University of \\ Al-Qadisiyah, Diwaniya, Iraq, ${ }^{2}$ Assistant lecturer, Department of Medical Microbiology, College of Medicine, \\ University of Al-Qadisiyah, Diwaniya, Iraq
}

\begin{abstract}
Background: Stroke is one of the leading causes of permanent disability worldwide. The most common cause of stroke is the occlusion of blood circulation by a thrombus (or embolism) although it can also be produced by the rupture of a vessel and subsequent bleeding in a certain region of the brain. Strokes can, therefore, be classified as ischemic or hemorrhagic.
\end{abstract}

Aim: The present study was conducted to investigate whether serum MIF levels are associated with severity in patients with ischemic stroke.

Method: A total of 40 patients with ischemic stroke were included in the study who were admitted to hospital from the period between March to August 2019, and other groups consist of 40 apparently healthy individuals. Two blood sample were taken from each patients one during attack (within 24 hours) and the other sample during follow up (within 1-3 months) for serum concentration of MIF by ELISA technique.

Results: Mean serum concentration of MIF in Ischemic stroke during attack (within 24 hours) was significantly higher than that of Ischemic stroke during follow up (within 1-3 months) and healthy control group; $17.094 \pm 2.25 \mathrm{ng} / \mathrm{ml}$ versus $12.83 \pm 4.16 \mathrm{ng} / \mathrm{ml}$ and $6.407 \pm 3.33 \mathrm{ng} / \mathrm{ml}$ respectively.

Keyword: Ischemic Stroke, MIF, NIHSS, ELISA.

\section{Introduction}

Stroke is one of the leading causes of permanent disability worldwide. The most common cause of stroke is the occlusion of blood circulation by a thrombus (or embolism) although it can also be produced by the rupture of a vessel and subsequent bleeding in a certain region of the brain. Strokes can, therefore, be classified as ischemic or hemorrhagic ${ }^{(\mathbf{1})}$. Ischemic stroke (IS) accounts for $85 \%$ of overall stroke and its pathophysiology are regulated by a combination of lifestyle, environmental, and unclear genetic risk factors ${ }^{(2)}$. Ischemic stroke is a heterogeneous multi-factorial, polygenic, complex disease resulting from the combination of vascular, environmental and genetic factors ${ }^{(3)}$.Ischemic Stroke often causes severe neurological and motor deficits, leading to decreased quality of life; stroke also has significant clinical and socioeconomic impacts ${ }^{(4)}$.
Annually, approximately 800,000 people in the United States have a stroke, and 130,000 die. It has accounted for nearly 5.7 million deaths worldwide, and $87 \%$ of these deaths occur in low and middle-income countries $^{(\mathbf{5})}$. Numerous studies have focused on the inflammatory reactions after stroke and identifying the roles of important inflammatory signaling molecules, mainly cytokines ${ }^{(5)}$. Cytokines are up-regulated in the brain after stroke and are expressed not only in immunological cells but also in glial cells and neurons ${ }^{(7)}$. However, the mechanisms leading to increased release of inflammatory cytokines in patients with stroke remain unclear. Macrophage migration inhibitory factor (MIF) a central cytokine of the innate immunity, includes 114 amino acid with a molecular weight of $12.5-\mathrm{kDa}$ and is expressed in a diversity of cell types, including $\mathrm{T}$ cells, macrophages, monocytes, endothelial cells and also in 
activated platelets ${ }^{(\mathbf{8})}$. Furthermore, it is recognized as a multifunctional cytokine participating in both immune and inflammatory responses ${ }^{(9)}$. MIF is up-regulated in the brain after cerebral ischemia and is involved in neuro-inflammation ${ }^{(\mathbf{1 0})}$. One study demonstrated that serum MIF levels at admission were positively correlated with infarct volume and long-term outcome in patients with acute ischemic stroke (AIS) $)^{(4)}$, while another study confirmed that elevated plasma levels of MIF at admission were associated with increased risk of post-stroke depression (PSD) in the next 3 months ${ }^{(11)}$.

\section{Materials and Method}

The current study was carried on 40 patients (18 males, 22 females) age range between 45-85 years from March to August 2019. Other groups consist of 40 apparently healthy individuals (20 male and 20 female) without any history of systemic disease were clinically considered as healthy also included in this study as a control group.We used NIHS Scale to determine the severity of the disease in ischemic stroke patients. We excluded patients with hemorrhagic stroke, stroke associated with surgery, severe trauma or organ ischemia. A six ml of blood samples were collected and withdrawn from each patient within 2 different period including three $\mathrm{ml}$ during attack (within 24 hours) and three $\mathrm{ml}$ during follow up (within 1-3 months) and three $\mathrm{ml}$ from healthy control by vein puncture using disposable syringes under aseptic technique were transferred to sterile Gel tube, and allow to clot at room temperature and centrifuge at $2500 \mathrm{rpm}$ for 10 minutes and the separated serum was saved in Eppendorf tubes and immediately frozen at -20 $\mathrm{C}$ till further use to avoid repeated thawing and freezing for MIF ELISA Kit (Elabscience USA) test. This study was in agreement with ethics of Al-Diwaniya Teaching
Hospital and verbal informed consent was obtained from all participants.

The National Institutes of Health Stroke Scale, or NIH Stroke Scale (NIHSS) is a tool used by healthcare providers to objectively quantify the impairment caused by a stroke. The NIHSS is composed of 11 items, each of which scores a specific ability between a 0 and 4 . For each item, a score of 0 typically indicates normal function in that specific ability, while a higher score is indicative of some level of impairment ${ }^{(\mathbf{1 2})}$. The individual scores from each item are summed in order to calculate a patient's total NIHSS score. The maximum possible score is 42 , with the minimum score being a $0^{(\mathbf{1 3})}$.

\section{Results}

Forty Ischemic stroke (IS) patients included in this study. The risk factors distribution of the study population are summarized in Table (1). There were no significant differences in smoking status as $(\mathrm{P}>0.05)$. The prevalence rates of hypertension, diabetes, alcoholism and heart disease were significantly increased in the IS group compared with those in the control group. Mean serum concentration of MIF in Ischemic stroke during attack (within 24 hours) was significantly higher than that of Ischemic stroke during follow up (within 1-3 months) and healthy control group; $17.094 \pm 2.25 \mathrm{ng} / \mathrm{ml}$ versus $12.83 \pm 4.16 \mathrm{ng} / \mathrm{ml}$ and $6.407 \pm 3.33 \mathrm{ng} / \mathrm{ml}$ respectively, $\mathrm{P}$-value was $(\mathrm{P}<0.0001)$, figure $(1)$. Table (2) showed highly significant correlation between serum levels of MIF and severity of ischemic stroke (NIHSS) $(\mathrm{P}<0.001$, $\mathrm{r}=0.512)$. Where serum levels of MIF increased with increasing severity of ischemic stroke as defined by the NIHSS score.

Table 1: Distribution of Ischemic Stroke patients and healthy control groups according to risk factors.

\begin{tabular}{|c|c|c|c|c|c|c|}
\hline \multirow{2}{*}{ Risk Factors } & \multicolumn{2}{|c|}{ Patients (IS) } & \multicolumn{2}{|c|}{ Healthy Control } & \multirow{2}{*}{ OR } & \multirow{2}{*}{$P$ value } \\
\hline & Positive N(\%) & Negative $\mathbf{N}(\%)$ & Positive N(\%) & Negative N(\%) & & \\
\hline Hypertension & $29(72.5)$ & $11(27.5)$ & $16(40)$ & $24(60)$ & 3.95 & $0.003(\mathrm{~S})$ \\
\hline Diabetes & $22(55)$ & $18(45)$ & $13(32.5)$ & $27(67.5)$ & 2.53 & $0.043(\mathrm{~S})$ \\
\hline Smoking & $17(42.5)$ & $23(57.5)$ & $15(37.5)$ & $25(62.5)$ & 1.23 & $0.648(\mathrm{NS})$ \\
\hline Alcoholism & $8(20)$ & $32(80)$ & $3(7.5)$ & $37(92.5)$ & 1.6 & $0.038(\mathrm{~S})$ \\
\hline Heart disease & $12(30)$ & $28(70)$ & $4(10)$ & $36(90)$ & 3.85 & $0.025(\mathrm{~S})$ \\
\hline
\end{tabular}

- NS : No significant association $(\mathrm{P}>0.05), \mathrm{S}$ : significant association $(\mathrm{P}<0.05)$. 


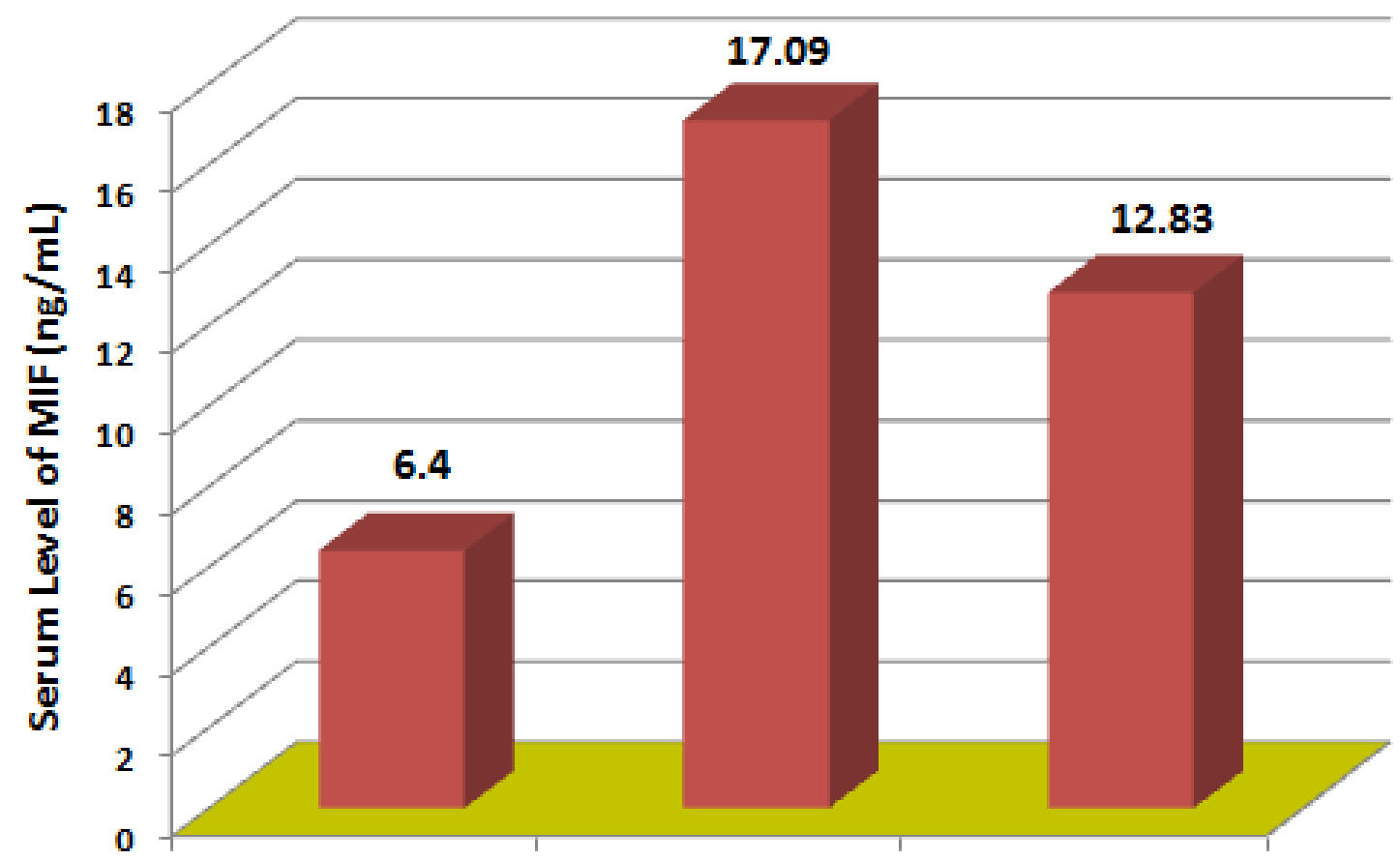

healthy control cases (stroke) during ettesk(stroke) during follow up

Figure (1): Comparison of serum concentration of MIF between healthy controls and patients with Ischemic stroke (during attack and during follow up).

Table (2): Correlation between MIF levels and Severity of Ischemic stroke (NIHS Scale).

\begin{tabular}{|c|c|c|c|c|c|}
\hline \multirow{2}{*}{$\begin{array}{l}\text { Serum MIF } \\
\text { conc.ng/ml }\end{array}$} & \multicolumn{3}{|c|}{ Severity of Ischemic stroke (NIHS Scale) } & \multirow{2}{*}{$\mathbf{P}$} & \multirow{2}{*}{$\mathbf{r}$} \\
\hline & Mild (1-4 Score) & Moderate (5-20 Score) & Severe (21-42 Score) & & \\
\hline Range & $\left(12.43 \_17.56\right)$ & $\left(10.737 \_19.60\right)$ & $\left(15.011 \_19.26\right)$ & \multirow{5}{*}{$\begin{array}{c}0.001 \\
(\mathrm{HS})\end{array}$} & \multirow{5}{*}{0.512} \\
\hline Mean & 14.98 & 16.891 & 18.229 & & \\
\hline SD & 4.33 & 3.12 & 2.45 & & \\
\hline SE & 0.39 & 0.52 & 0.42 & & \\
\hline $\mathrm{N}$ & 7 & 17 & 16 & & \\
\hline
\end{tabular}

- $\mathrm{HS}=$ highly significant association $(\mathrm{P}<0.01)$.

\section{Discussion}

The current study showed that $29(72.5 \%)$ of the patients with ischemic stroke had hypertension, and these results indicate high significantly increased in patients with ischemic stroke compared with those in the healthy control group $(\mathrm{P}=0.003, \mathrm{OR}=3.95)$ table (1). This leads us to believe that hypertension is highly nondiagnosed in population due to the lack of an active noncommunicable disease screening program, failure to take routine blood pressure measurements, and a general lack of awareness among health practitioners about hypertension and their complications ${ }^{(\mathbf{1 4})}$. The present results are generally in accordance with previous studies where indicated that hypertension reduction being associated with a reduced rate of ischemic stroke ${ }^{(\mathbf{1 5})}$. These results consistence with results of $\mathrm{Li}$ et $\mathrm{al}^{(4)}$, who found that $91(62.3 \%)$ with hypertension and indicate that hypertension significantly increased in the ischemic stroke group compared with those in the control group. In our results there are $22(55 \%)$ of patients with ischemic stroke had diabetes, and these results indicate 
significant correlation between diabetes and ischemic stroke when compared with healthy controls groups ( $\mathrm{p}$ $<0.05, \mathrm{OR}=2.53$ ). Diabetes is a recognized independent risk factor for stroke and is associated with higher morbidity and mortality ${ }^{(\mathbf{1 6})}$. The physiology underlying the increased risk of ischemic stroke in diabetics may be attributable to the increased prevalence of intracranial stenosis in this population ${ }^{(17)}$. Vasculopathy induced by chronic diabetes related endothelial damage results in acceleration of atherosclerosis inherent to diabetes ${ }^{(\mathbf{1 8})}$. Which in consistence with old studies that showed diabetes can commonly causes small infarcts which less likely to lead to a fatal stroke ${ }^{(19)}$.

Although our results indicate 17(42.5\%) from individuals with ischemic stroke are active smokers, but these results shows there is no significant correlation between the active smokers and ischemic stroke when compared with control groups, although the smokers are high in ischemic stroke groups $(\mathrm{p}>0.05, \mathrm{OR}=1.23)$ table(1). Although our study indicate non-significant association between the smokers and ischemic stroke when compared with control groups, but remain the smoking as important risk factors for ischemic stroke. The mechanism by which passive smoking can increase the risk of stroke has been reported in many studies. Passive smoking can lead to carotid atherosclerosis ${ }^{(\mathbf{2 0})}$, and the levels of homocysteine, fibrinogen, and oxidized low-density lipoprotein cholesterol can also be elevated by smoking ${ }^{(21)}$. About 8 (20\%) of patients with ischemic stroke were alcohol consumption, as table (1), and there is a significant correlation between the ischemic stroke and alcoholism when compared with healthy control group $(\mathrm{P}=0.038, \mathrm{OR}=1.6)$. Ischemic stroke is caused by a number of different pathophysiological mechanisms and alcohol drinking might have contrasting effects on ischemic stroke. For example, moderate and high alcohol consumption is associated with an elevated risk of atrial fibrillation, which is a risk factor for ischemic stroke $^{(22)}$. The risk for ischemic stroke was significantly higher for binge drinkers than for subjects with no heavy drinking pattern $^{(23)}$. The present results consistence with that of $\mathrm{Xu}$ et $\mathrm{al}^{(\mathbf{1 1})}$, who found that $52(15.6 \%)$ with alcohol consumption.

The frequency of heart disease among the ischemic stroke patients are $12(30 \%)$ as in table (3-5), and these results indicate significant association between heart disease and ischemic stroke when compared with healthy control groups $(p<0.05, O R=3.85)$. Previous study showed that approximately $10 \%$ to $24 \%$ of patients with stroke have heart failure ${ }^{(24)}$. Our study indicate frequency lower than Yalcin et al ${ }^{(25)}$, which showed (57.5\%) of the stroke patients had history of ischemic heart disease, and it may be due to short longevity of our patients after developing ischemic heart attack as compared to others in the developed countries, and short longevity after developing coronary heart disease. Furthermore, increasing evidence that heart disease is independently associated with cognitive impairment exists; and cognitive impairment is associated with decreased survival among hospitalized stroke patients ${ }^{(24)}$.

Figure (1) showed that mean serum concentration of MIF were highly significantly increased among cases with ischemic stroke during attack (within 24 hours) $17.094 \pm 2.25 \mathrm{ng} / \mathrm{ml}$ as compared to apparently healthy controls $6.407 \pm 3.33 \mathrm{ng} / \mathrm{ml}$, these results revealed a significant association between the concentration of MIF and the ischemic stroke $(\mathrm{P}<0.001)$. A study by Inacio et $\mathrm{al}^{(\mathbf{1 0})}$, (2011), indicated that MIF promotes neuronal death and aggravates neurological deficits after experimental stroke. They found that MIF promoter activity was significantly upregulated by hypoxia during ischemic stroke and that MIF protected neurons against oxygen-glucose deprivation. Thus, the reason why serum MIF levels are increased in ischemic stroke patients remains unknown. Serum MIF levels were found to be decreased with blood-sampling time, with the highest levels of serum MIF at ischemic stroke during attack (within 24 hours) $(17.094 \pm 2.25 \mathrm{ng} / \mathrm{ml})$, and this levels decrease at cases with ischemic stroke during follow up(within 1-3 months) $(12.83 \pm 4.16 \mathrm{ng} / \mathrm{ml})$, these difference in serum MIF levels between ischemic stroke during attack (within 24 hours) and ischemic stroke during follow up(within 1-3 months) was statistically significant $(\mathrm{P}<0.001)$, figure (1). These results indicate that MIF might play an important role in inflammatory process of central nervous system. However, serum MIF levels were highly significantly elevated in any time of ischemic stroke when compared with the controls ( $\mathrm{P}$ $<0.001$ ). Table (2) showed positive correlation between serum MIF levels and severity of ischemic stroke (NIHSS) $(\mathrm{P}<0.001, \mathrm{r}=0.512)$. where serum levels of MIF increased with increasing severity of ischemic stroke as defined by the NIHSS score, were highest MIF levels in the severe group $(18.229 \pm 2.45 \mathrm{ng} / \mathrm{ml})$ as compared to moderate and mild severity $(16.891 \pm 3.12 \mathrm{ng} / \mathrm{ml}$ and $14.98 \pm 4.33 \mathrm{ng} / \mathrm{ml}$ respectively). The present results are consistence with results of $\mathrm{Xu}$ et al ${ }^{(\mathbf{1 1 )}}$, which indicated there was a positive correlation between levels of MIF and the NIHSS $(r=0.248, \mathrm{P}<0.001)$. 


\section{Conclusions}

Our study demonstrated that the serum level of MIF significantly increased following ischemic stroke. The serum MIF levels at admission were positively correlated with the infarct volumes and the severity of patients with IS. In this study we found increased inflammatory response after ischemic stroke compared with healthy control, represented by increased levels of MIF.

Financial Disclosure: There is no financial disclosure.

Conflict of Interest: None to declare.

Ethical Clearance: All experimental protocols were approved under the Department of Medical Microbiology and all experiments were carried out in accordance with approved guidelines.

\section{References}

1. Lees KR, Bluhmki E, von Kummer R. Time to treatment with intravenous alteplase and outcome in stroke: an updated pooled analysis of ECASS, ATLANTIS,NINDS, and EPITHET trials, Lancet . 2010; 375 (9727): 1695-1703.

2. Della-Morte D, Guadagni F, Palmirotta R. Genetics of ischemic stroke, stroke-related risk factors, stroke precursors and treatments. Pharmacogenomics.2012; 13:595-613.

3. Kim SK, Kang SW,Kim DH. Matrix metalloproteinase-3 gene polymorphisms are associated with ischemic stroke.J. Interferon Cytokine Res. Off. J. Int. Soc. Interferon Cytokine Res. 2012; 32: 81-86.

4. Li YS, Chen W, Liu S. Serum macrophage migration inhibitory factor levels are associated with infarct volumes and long-term outcomes in patients with acute ischemic stroke[J]. International Journal of Neuroscience. 2017; 127(6): 539-546.

5. Feigin VL, Krishnamurthi R, Mensah GA. Global and regional burden of stroke during 1990-2010: Findings from the Global Burden of Disease Study 2010. Lancet. 2014; 383:245-54.

6. Ormstad, H.; Aass, HC.; Lund-Sørensen, N.; et al. (2011): Serum levels of cytokines and C-reactive protein in acute ischemic stroke patients, and their relationship to stroke lateralization, type, and infarct volume. J Neurol. 258:677-85.

7. Vila N, Castillo J, Dávalos A. Proinflammatory cytokines and early neurological worsening in ischemic stroke. Stroke. 2000; 31:2325-9.

8. Chatterjee M, Borst O, Walker B. Macrophage migration inhibitory factor (MIF) limits activationinduced apoptosis of platelets via CXCR7dependent Akt signaling. Circ Res.2014; 115:939949.

9. Liu YC, Tsai YH, Tang SC. Cytokine MIF enhances blood-brain barrier permeability: impact for therapy in ischemic stroke. Sci Rep.2018; 8(1):743.

10. Inacio AR, Ruscher $\mathrm{K}$, Leng L. Macrophage migration inhibitory factor promotes cell death and aggravates neurologic deficits after experimental stroke. J Cereb Blood Flow Metab: official Journal of the international Society of Cerebral Blood Flow and Metabolism.2011; 31: 1093-1106.

11. Xu T, Pu S, Ni Y. Elevated plasma macro-phage migration inhibitor factor as a risk factor for the development of post-stroke depression in ischemic stroke. J Neuroimmunol.2018; 320:58-63.

12. National Institute of Health, National Institute of Neurological Disorders and Stroke. Stroke Scale.

13. Hage V. The NIH stroke scale: a window into neurological status". Nursing Spectrum. 2011; 24 (15): 44-49.

14. Gebremariam SA, Yang HS. Types, risk profiles, and outcomes of stroke patients in a tertiary teaching hospital in northern Ethiopia. ENeurologicalSci.2016; 3:41-47.

15. Arima H, Chalmers J, Woodward M. Lower target blood pressures are safe and effective for the prevention of recurrent stroke: the PROGRESS trial. J Hypertens.2006; 24:1201-1208.

16. Zhu S, McClure LA, Lau H. Recurrent vascular events in lacunar stroke patients with metabolic syndrome and/or diabetes. Neurology. 2015;85:935-941.

17. PalacioS, McClure L, Benavente O. Lacunar strokes in patients with diabetes mellitus: risk factors, infarct location, and prognosis: the Secondary Prevention of Small Subcortical Strokes Study. Stroke.2014; 45:2689-2694.

18. Tun NN, Arunagirinathan G, Munshi, SK. Diabetes mellitus and stroke: A clinical update. World J Diabetes.2017; 8(6): 235-248.

19. Arboix A, Rivas A,Eroles L. Cerebral infarction in diabetes: Clinical pattern, stroke subtypes, and 
predictors of in hospital mortality. MC Neurol. 2005; 5: 9.

20. Rehill N, Beck CR, Yeo KR. The effect of chronic tobacco smoking on arterial stiffness. Br J Clin Pharmacol.2006; 61:767- 73.

21. Hackshaw A, Morris JK, Boniface S. Low cigarette consumption and risk of coronary heart disease and stroke: meta-analysis of 141 cohort studies in 55 study reports. BMJ .2018; 5855 -69.

22. Larsson SC, Drca N, Wolk, A. Alcohol consumption and risk of atrial fibrillation: a prospective study and dose-response meta-analysis. J Am Coll Cardiol. 2014; 64:281-289.
23. Sundell L, Salomaa V, Vartiainen E. Increased Stroke Risk Is Related to a Binge Drinking Habit. aha journals.org. 2008; 3179-3184.

24. Divani AA, Vazquez G, Asadollahi M. Nationwide frequency and association of heart failure on stroke outcomes in the United States. J Card Fail. 2009; 15:11-16.

25. Yalcin E, Ylacin M, Celik Y. Risk Factors for Recurrent Ischemic Stroke in Turkey. Medical Journal of Trakya University. 2008; 25: 117-123. 